\title{
Tiers garant oder Tiers payant?
}

\section{Ernst Gähler ${ }^{a}$, \\ Kerstin Schutz ${ }^{b}$}

a Dr. med., Vizepräsident der FMH, Departementsverantwortlicher Ambulante Tarife und Verträge Schweiz

b FMH, Abteilung Ambulante Tarife und Verträge Schweiz

Mit Unterstützung von: Dr. med. Peter Wiedersheim, Co-Präsident KKA Anton Prantl, Direktionspräsident Ärztekasse, Peter Frutig, CEO PonteNova, Renato Laffranchi, Dr. sc. nat. General Manager NewIndex
Korrespondenz: FMH Abteilung Ambulante Tarife und Verträge Frohburgstrasse 15 $\mathrm{CH}-4600$ Olten

tarife.ambulant[at]fmh.ch
Auf die Frage, welchem Abrechnungsverfahren im ambulanten Bereich der Vorzug zu geben ist, scheiden sich seit deren Einführung die Geister. Eine schematische Darstellung beider Abrechnungsverfahren findet sich in Abbildung 1.

In den vergangenen Monaten hat sich der Diskurs intensiviert und im Januar 2014 hat sich die FMH mit einem Positionspapier [1] sowie einem Artikel in der Schweizerischen Ärztezeitung [2] für den Status quo und damit für den Tiers garant als Standardmodell ausgesprochen.

\section{Kein «Lackmustest»}

Diese Positionierung erfolgt aus dem Bestreben, die vom Gesetzgeber gegebenen Optionen und damit die bestehende Wahlfreiheit [3] beizubehalten: Der Tiers garant ist als Regelfall definiert, von dem allerdings Leistungserbringer und Versicherer abweichen dürfen. Der Tiers payant ist im ambulanten Bereich daher nicht ausgeschlossen. Die Ärztin / der Arzt (nachfolgend Arzt) entscheidet, wann er vom gesetzlich definierten Normalfall abweicht [4]. Beide Systeme haben ihre Daseinsberechtigung - jeder Arzt wählt das System, welches am besten seiner individuellen Situation entspricht.

Aus Sicht der FMH ist der Tiers garant für den Normalfall prädestiniert. Zu den wichtigsten Gründen hierfür zählen:
- Der Patientin / dem Patienten (nachfolgend der Patient) wird eine bedeutendere und aktivere Rolle zuteil, als im Tiers payant. Er kann selbst entscheiden, welche Rechnungen er seinem Versicherer zur Vergütung einreicht und damit zur Kenntnis bringt.

- Das System Tiers garant schafft volle Transparenz des Arztes gegenüber dem Patienten und seine Daten werden geschützt.

- Der Tiers garant trägt zu Kosteneinsparungen der Sozialversicherungen bei und hilft so, unnötige Prämiensteigerungen zu vermeiden.

In der Ausgabe 18 der Schweizerischen Ärztezeitung vom 30. April 2014 reflektiert Dr. Franz Marty Entwicklungen der letzten 20 Jahre und kommt nach seinen Ausführungen zu einem anderen Schluss: «Der Tiers garant zeigt [gegenüber dem Tiers payant] gravierende Nachteile.»

Der folgende Abschnitt nimmt seine Argumente noch einmal auf und zeigt, wo Gemeinsamkeiten zu finden und worin Unterschiede zu begründen sind.

\section{Ausgrenzung von Patienten im Tiers garant?}

Die Wahl des Abrechnungssystems hat nichts mit der Art der Behandlung zu tun. Im Tiers garant ist der Patient Rechnungsempfänger und begleicht die Arzt-

\section{Tiers garant}

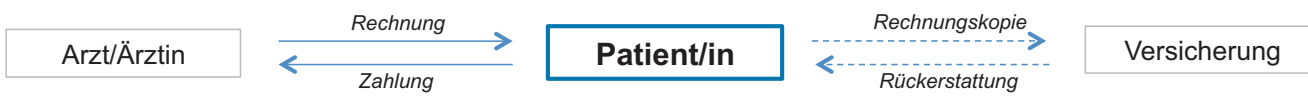

\section{Tiers payant}

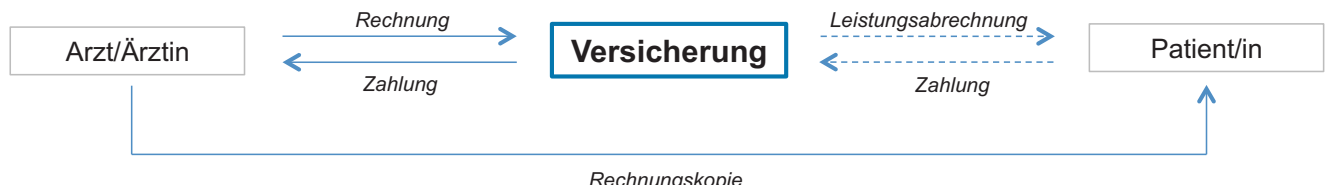

Rechnungskopie

Abbildung 1

Schematische Darstellung der Abrechnungsverfahren Tiers garant und Tiers payant. 
rechnung. Er muss jedoch keinen Vorschuss leisten: Ist die Rechnung für eine sofortige Begleichung durch den Patienten zu hoch, wartet er bis er den Rückforderungsbetrag von der Krankenkasse erhalten hat und zahlt erst anschliessend den Rechnungsbetrag.

Diese Aussage wird auch seitens verschiedener Versicherer gestützt: Im April 2013 schreibt die Allianz Schweizer Krankenversicherer (heute curafutura): «Es liegt im Interesse jedes einzelnen Anbieters, seinen Versicherten den best- und schnellstmöglichen Rückerstattungsservice zu bieten. So hat die versicherte Person durchaus die Möglichkeit, die Arztrechnung erst nach erfolgter Rückerstattung durch den Krankenversicherer zu begleichen und muss keine Vorkasse leisten. [...] Keinem Mitglied unserer Gesellschaft wird der Zugang [zum schweizerischen Gesundheitssystem] verwehrt.» [5]. Voraussetzung ist, dass der Patient im Vorfeld darüber informiert wird - beispielsweise beim Abschluss seiner Krankenversicherung oder auch durch das Gespräch mit seinem Arzt.

\section{Höherer Aufwand für das Mahnwesen im Tiers garant?}

Im Durchschnitt gibt es im Tiers payant als auch im Tiers garant bei circa 3\% der Rechnungen Zahlungsverzögerungen [6]. Das bedeutet, dass auch im Tiers payant - beispielsweise durch Rückweisungen von Versicherungen - der Arztpraxis ein administrativer Aufwand entsteht, der mit demjenigen im Tiers garant vergleichbar ist.

Weiter zeigen Auswertungen der Ärztekasse [7], dass sich die Höhe der Zahlungsausfälle in beiden Abrechnungsverfahren nicht unterscheiden und je nach Kanton im Tiers garant sogar geringer ausfallen. Trustcenter und die Ärztekasse bieten Dienstleistungen an, die den Rechnungsversand und das Mahnwesen der Arztpraxen deutlich erleichtern.

Entscheidet sich eine Arztpraxis für die Abrechnung via Tiers payant, ist sie dennoch gesetzlich verpflichtet, dem Patienten eine Kopie der Rechnung zukommen zu lassen, eine Einsparung ergibt sich damit nicht.

\section{Aufwendige Abtretungserklärungen}

Die Abtretungserklärung [8] bietet die Möglichkeit den Patienten im Ausnahmefall vor hohen Rechnungen zu schützen. Sie gibt anderseits dem Arzt die Sicherheit, wenn er befürchtet, dass Rechnungen, trotz garantierter Rückvergütung durch die Kasse an den Patienten, nicht bezahlt werden. Der Aufwand dafür ist, auch aus Sicht der FMH, zu optimieren. Der Versand einer Abtretungserklärung pro gestellte Rechnung ist zu aufwendig und könnte vereinfacht werden. Der Vorteil der Abtretungserklärung liegt aber klar darin, dass die Möglichkeit besteht, diese zusammen mit dem Patienten für einen bestimmten Zeitraum vereinbaren zu können.
Der Patient hat also heute schon Einfluss auf die Wahl des Abrechnungssystems.

Marty resümiert: «Die Wahl des Abrechnungsmodus soll freigegeben werden. Der Patient soll über den Abrechnungsmodus entscheiden können.» [9]. Zugegebenermassen erscheint dieser Vorschlag zunächst naheliegend und plausibel - es ist schliesslich der Patient, der im Mittelpunkt steht. Doch der Arzt ist derjenige, der eine Leistung erbringt und quasi so Vorkasse leistet. Sollte es daher nicht auch ihm überlassen sein, welche Abrechnungsform er wählt?

\section{Einbindung in den elektronischen Verarbeitungsprozess}

Die FMH ist klar der Meinung, dass Entwicklungen im Bereich eHealth nicht abhängig sein dürfen vom gewählten Abrechnungssystem. Eine optimale Verfügbarkeit von statistischen Daten zu verschiedenen Zwecken wird heute von unterschiedlichen Seiten gefordert. Das Ziel, die ärzteeigene Datensammlung via Trustcenter zu stärken, kann mit beiden Abrechnungsverfahren erreicht werden: Trustcenter bieten ihren Mitgliedern die Übermittlung der Daten sowohl im Tiers garant als auch im Tiers payant an [10]. Auch Krankenversicherer bestätigen, dass die Verarbeitung von Rechnungen im Tiers garant schon heute in nahezu identischer Qualität möglich ist [11].

\section{Verständliche Rechnungen und Rechnungskontrolle}

Grundsätzlich ist der Patient derjenige, der die Rechnung des Arztes am besten überprüfen kann, da er ausser dem Arzt als einziger weiss, welche Leistungen erbracht worden sind.

Die Voraussetzung ist in beiden Systemen gegeben, sofern der Patient die Rechnung erhält. Der Kritik von Franz Marty, dass die Rechnungen für Patienten oftmals nicht lesbar sind, ist sich die FMH bewusst. Sie arbeitet im Rahmen der Gesamtrevision TARMED mit ihren Partnern deshalb daran, die Bezeichnungen der Tarifpositionen zu vereinfachen und prüfen weitere Möglichkeiten, das Rechnungsformular im Sinne des Patienten lesbarer und verständlicher zu gestalten.

\section{Verwaltungsaufwand}

Auswertungen der Ärztekasse zeigen, dass bei der flächendeckenden Einführung des Tiers payant das Rechnungsvolumen um ca. 750 Millionen Franken steigen würde: Diese Befürchtung teilt Dr. Marty nicht. Doch auch Versicherer bestätigen, dass rund 15 Prozent aller Arztrechnungen für ambulante Behandlungen von Versicherten nicht an ihre Krankenkassen weitergeleitet werden und damit Kosten gespart werden [12]. Der Verwaltungsaufwand der Krankenversicherer würde im Tiers payant steigen, ohne zusätzlichen Nutzen zu generieren. Hinzu kommt, dass der Patient eine unnötige Leistungsabrechnung 
der Versicherung erhält, für Kosten, die er sowieso selbst begleichen muss. Weiterhin ist im Tiers payant zu bedenken, dass für alle Nichtpflichtleistungen dem Patienten ohnehin eine separate Rechnung ausgestellt werden muss. Im Tiers garant genügt in der Regel eine Rechnung [13].

Der höhere Verwaltungsaufwand verursacht Mehrkosten, die sich bei einem schweizweiten Umstieg auf das System des Tiers payant in jedem Fall auf Prämien und/oder Taxpunktwerte auswirken würden. Die erwähnten Mehrkosten entsprächen keiner realen Kostensteigerung!

\section{Datenschutz}

Bereits mehrmals erwähnt wurde der Datenschutz des Patienten, der in die Überlegungen von Dr. Marty keinen Eingang findet. Dem Daten- sowie dem Persönlichkeitsschutz des Patienten wird unter heutigen Bedingungen nur im Tiers garant vollständig Folge geleistet. Der Patient entscheidet selbst, welche Rechnungen die Kasse erhält, und hat es damit in der Hand, welche Informationen der Versicherer über Veranlagungen, Krankheiten oder Eingriffe erhält.

\section{Fazit}

Weder der Tiers garant noch der Tiers payant stürzen die Ärzte ins Verderben. Doch unter den heutigen Voraussetzungen ergibt sich eine Situation, die aus Sicht der FMH und ihren Partnern für die Beibe-

\section{Interaktiver Artikel}

Wollen Sie diesen Artikel kommentieren? Nutzen Sie dafür die Kommentarfunktion in der OnlineVersion oder sehen Sie nach, was Ihre Kolleginnen und Kollegen bereits geschrieben haben: www.saez.ch/aktuelle-ausgabe/interaktive-beitraege/ haltung des Tiers garant als Standard-Abrechnungsmethode im ambulanten Bereich spricht. Diese Voraussetzungen sind jedoch stets zu überprüfen und die Systeme anhand aktueller Situation zu optimieren oder zu überdenken.

\section{Referenzen}

1 www.fmh.ch/files/pdf14/20130730_Positionspapier FMH_Tiers_garant_D.pdf

2 Gähler E, Marty I, Schutz K. Die FMH für den Tiers garant. Schweiz Ärztezeitung. 2014;95(5):147-9. www.fmh.ch/files/pdf14/SAEZ_6_Tiers_garant_D.pdf

3 Art. 42 KVG; Zum Teil auch parlamentarische Vorstösse fordern einen generellen Wechsel zum Tiers payant auch im ambulanten Bereich. Vgl. Parlamentarische Initiative des Mouvements Citoyens Romand, www.parlament.ch/d/suche/seiten/geschaefte. aspx?gesch_id=20110492

\section{Art. 42 KVG Abs. 1, 2}

5 www.ask-aams.ch/index.php/de/aktuell/ inside-1-august-2012/93-deutsche-kategorien/news/ newsletter/187-tiers-garant-versus-tiers-payant

6 Daten Ärztekasse.

7 Auswertungen verschiedener Kantone im FMH Positionspapier S.4. www.fmh.ch/files/ pdf14/20130730_Positionspapier_FMH_Tiers_ garant_D.pdf

8 Mit einer Abtretungserklärung (KVG Art. 42 Abs. 1) kann der Patient zusammen mit dem Arzt vorübergehend vereinbaren, dass die Rechnung direkt vom Arzt an die Krankenkasse gesendet wird.

9 Marty F. Stürzt der Tiers payant die Ärzte ins Verderben? Schweiz Ärztezeitung. 2014;95(18):710-2.

10 Erich Honegger. Bulletin HKO Nr. 6, S.3.

11 Christoph Bangerter. Punktuell, Newsletter der PonteNet 5/13.

12 www.ask-aams.ch/index.php/de/aktuell/ inside-1-august-2012/93-deutsche-kategorien/news/ newsletter/187-tiers-garant-versus-tiers-payant

13 Gemäss KVV, Art. 59 Abs. 2 müssten die Rechnungen generell gesplittet werden, doch wird in der Praxis in der Regel eine Rechnung akzeptiert. 